\title{
Poly $r(C)$ Binding Protein-1 is Central to Maintenance of Cancer Stem Cells in Prostate Cancer Cells
}

\author{
Qi Chen Zhi-kang Cai Yan-bo Chen Meng Gu Da-chao Zheng Juan Zhou \\ Zhong Wang
}

Department of Urology and Andrology, Ninth People's Hospital, School of Medicine, Shanghai Jiaotong University, Shanghai, China

\section{Key Words}

Poly $\mathrm{r}(\mathrm{C})$ binding protein-1 $-\mathrm{PCBP} 1 \cdot$ Prostate cancer $\bullet$ Stemness $\cdot$ Metastasis $\bullet$ Cancer stem cells

\begin{abstract}
Aims: To investigate global proteomic changes induced in CD44+CD24- stem cells isolated from the prostate cancer cell lines, LNCaP and DU145, post prolonged TGF- $\beta$ treatment in order to understand underlying mechanisms that promote stemness in prostate cancer cells. Methods: $C D 44+C D 133+\alpha 2 \beta 1$ Integrin $+C D 24-$ population was isolated from mock or TGF- $\beta$ treated (7 days) prostate cancer cell line, LNCaP, through fluorescent activated cell sorting. Cell lysates were obtained from the \pm TGF- $\beta$ cell population and proteomics profiling (MS/MS) was performed by mass spectrometry. Relative enrichment or depletion in the CD44+CD24population post-TGF- $\beta$ treatment was determined relative to mock-treated CD44+CD24- cells post normalization to GAPDH expression levels. Results obtained from MS/MS were validated using immunoblotting. Functional validation of one putative regulator was performed using gain-of-function strategy to investigate its role in rendering stemness in LNCaP and DU145 cells in vitro and in promoting tumorigenicity in vivo. Results: TGF- $\beta$ treatment caused significant enrichment of CD44+CD24- population in LNCaP cells $(22.35 \pm 0.94 \%$ in mock treated vs $95.23 \pm 2.34 \%$ in TGF- $\beta$ treated cells; $P<0.01$ ), which were also positive for CD133 and $\alpha 2 \beta 1$ Integrin. Mass spectrometry analysis of the enriched cell population revealed that sixty-three proteins were either up- or down-regulated greater than five folds, out of which the poly $r(C)$ binding protein (PCBP)- 1 was the most down-regulated (9.31 \pm 0.05 folds). Ectopic overexpression of PCBP1 in LNCaP and DU145 cells not only attenuated enrichment of $C D 44+C D 133+C D 24-$ population in these cells following TGF- $\beta$ treatment, but also significantly decreased tumorigenicity of the stem cell subset, as assessed by in vitro soft agar colony formation and in vivo xenograft assays. Conclusion: Our proteomic profiling and subsequent validation indicate that PCBP1 is central to CSCs enrichment and functionality in prostate cancer.

Q. Chen, Z.-k. Cai and Y.-b. Chen these authors contributed equally to this article.

Zhong Wang, MD., PhD.

Department of Urology and Andrology, Ninth People's Hospital, School of Medicine, Shanghai Jiaotong University. 639 Zhi Zaoju Road, Shanghai, 200011 (China) Tel. +86-13301980998, Fax +86-21-23271699, E-Mail zhongwang2010@sina.com
\end{abstract}

KARGER 125 


\section{Introduction}

Prostate cancer (PCa) is the second leading cause of cancer mortality and is the one that has shown the maximum increase of incidence in the last decade (World Health Organization, 2012). Despite the intensive and extensive studies on its cause, early detection, and therapy, there are still many unanswered questions about the molecular mechanisms underlying the uncontrolled expansion of PCa cells, as well as tumor recurrence and metastasis, the latter being attributed to be responsible for $90 \%$ of the deaths associated with PCa. Emerging evidence suggest that a minor population of cells in prostate tumors with stem cell properties called cancer stem cells (CSCs) driving metastatic progression, recurrence and resistance to different treatment regimens [1-4].

CSC populations may be phenotypically purified based on the expression of cell surface markers like CD44, CD34, CD133, and epithelial cell adhesion molecule (EpCAM) [5, 6]. CD44 has been widely used for enriching the stem cell populations in cancers of the prostate $[7,8]$. Alongside, several PCa cell populations with enhanced tumor-initiating capacities that fit the definitions of CSCs have been identified [8-11]. The CD44+ PCa cells have been shown to express higher levels of 'stemness' genes including Oct-3/4, Bmi- $1, \beta$ - catenin, and Smoothened [8]. Clonal analysis have indicated that a small subset of the CD44+ cells can undergo asymmetric cell division, with only one of the daughter cells retaining CD44 expression [8].

It has been elucidated by various research groups that TGF- $\beta$ secreted from the tumor microenvironment and bone marrow derived mesenchymal cells both contribute to stemness of prostate cancer cells $[12,13]$. However, not much, if any, is known about the underlying mechanisms that contribute to the maintenance of PCa stem cell population in the presence of secreted TGF- $\beta$. Hence, the objective of the current study was to investigate global proteomic changes induced in CD44+CD133+CD24- stem cells isolated from the prostate cancer cell lines, DU145 and LNCaP, post prolonged TGF- $\beta$ treatment in order to understand underlying mechanisms that promote stemness in prostate cancer cells.

\section{Materials and Methods}

\section{Cell lines and reagents}

This study was carried out in strict accordance with the recommendations in the Guide for the Care and Use of Laboratory Animals of the National Institutes of Health. The protocol was approved by the Committee on the Ethics of Animal Experiments of Shanghai Jiaotong University. All surgery was performed under sodium pentobarbital anesthesia, and all efforts were made to minimize suffering. LNCaP and DU145 cell lines were purchased from ATCC (Manassas, VA). LNCaP cells were grown in RPMI 1640 supplemented with 10\% fetal bovine serum, $2 \mathrm{mM}$ L-glutamine, 1\% HEPES, 1\% sodium-pyruvate and 10 $\mathrm{ml} / \mathrm{L}$ of $100 \times$ antibiotic-antimycotic solution (Sigma-Aldrich, St. Louis, MO). DU145 cells were grown in DMEM supplemented with $10 \%$ fetal bovine serum and $10 \mathrm{ml} / \mathrm{L}$ of $100 \times$ antibiotic-antimycotic solution. Cells were maintained in a humidified atmosphere $\left(5 \% \mathrm{CO}_{2}\right)$ at $37^{\circ} \mathrm{C}$. Where indicated, LNCaP and DU145 cells were treated with TGF- $\beta 1$ (R\&D Systems, Minneapolis, MN) at a final concentration of $5 \mathrm{ng} / \mathrm{ml}$ for up to 7 days. The anti-PCBP1 and anti-GAPDH antibodies were purchased from Abcam (Cambridge, MA). Western blot reagents were purchased from Amersham Pharmacia Biotech (Piscataway, NJ).

\section{Fluorescence-activated cell sorting}

Anti-CD24-APC and anti-CD44-PE antibodies were purchased from BD Biosciences. Anti-CD133Biotin and anti-Alpha-2 Beta-1 ( $\alpha 2 \beta 1$ ) Integrin-FITC antibody were purchased from Miltenyi Biotech and Abcam, respectively. Cell labeling with fluorescent-conjugated antibodies was performed according to the manufacturer's recommendations. Sorting of antibody-labeled cells was carried out on a FACSAria cell sorter (BD Biosciences). Sorted CD24 ${ }^{-}$D $44^{+}$cells from LNCaP or DU145cells were cultured in MEM containing $10 \%$ fetal bovine serum and insulin $(10 \mu \mathrm{g} / \mathrm{ml})$ at $37^{\circ} \mathrm{C}$ with $5 \% \mathrm{CO}_{2}$. Sorted CD $24^{-\mathrm{CD}} 44^{+}$and $\mathrm{CD} 24^{+} \mathrm{CD} 44^{-}$ cells were subsequently tested for expression of CD133 and $\alpha 2 \beta 1$ Integrin. 


\section{Quantitative Real-Time RT-PCR (qRT-PCR)}

qRT-PCR was performed using an Applied Biosystems Prism 7300 Sequence Detection System with SYBR Green PCR Master Mix (Applied Biosystems, Inc., Foster City, CA, USA). Primers were designed to be specific for CD44 variants:

CD44s: forward primer 5'-TCC AAC ACC TCC CAG TAT GAC A-3' and reverse primer 5'-GGC AGG TCT GTG ACT GAT GTA CA-3';

CD44v3: forward primer 5'-GCA GGC TGG GAG CCA AAT-3' and reverse primer 5'-GAG GTG TCT GTC TCT TTC ATC TTC ATT-3';

CD44v6: forward primer 5'-GGA ACA GTG GTT TGG CAA CAG-3' and reverse primer 5'-TTG GGT GTT TGG CGA TAT CC-3';

CD44v10: forward primer 5'-CAG GTG GAA GAA GAG ACC CAA A-3' and reverse primer 5'-GGA TGA AGG TCC TGC TTT CCT T-3'.

CD133 forward and reverse primers used were 5'-GGA CCC ATT GGC ATT CTC-3' and 5'-CAG GAC ACA GCA TAG AAT AAT C-3'; CD24 forward and reverse primers were 5'-TGC TCC TAC CCA CGC AGA TT-3' and 5'-GGC CAA CCC AGA GTT GGA A-3'.

Amplification data were analyzed by 2- $\Delta \mathrm{CT}$ method with an Applied Biosystems Prism Sequence Detection Software (Version 2.1). The cycle threshold (CT) value corresponding to the PCR cycle number at which fluorescence emission in real time reached a threshold above the baseline emission was determined for each gene of interest and normalized to a cycle threshold for a housekeeping gene (TBP) determined in parallel. All qRT-PCR reactions were performed in triplicate and three independent sets of samples were used in each experiment. Data is represented as means \pm standard deviation (SD).

\section{Mass spectrometry and data analysis}

Sorted CD44+CD24- cells from mock or TGF- $\beta$ treated (for 7 days) LNCaP cells in triplicate were lysed using NET buffer [50 mmol/L Tris-HCl, (pH 7.4), $150 \mathrm{mmol} / \mathrm{L} \mathrm{NaCl}, 0.1 \%$ NP40, $1 \mathrm{mmol} / \mathrm{L}$ EDTA, 0.25\% gelatin, $0.02 \%$ sodium azide, $1 \mathrm{mmol} / \mathrm{L}$ phenylmethylsulfonyl fluoride, and $1 \%$ aprotinin]. The lysates were centrifuged at $15,000 \times g$ for 30 minutes at $4^{\circ} \mathrm{C}$. Collected supernatant was dialyzed against PBS, proteins were reduced with $5 \mathrm{mM}$ Tris 2-carboxyethyl phosphine (TCEP) and alkylated with $10 \mathrm{mM}$ iodoacetamide. Samples were digested with trypsin (Promega) in a 1:50 ratio for $12 \mathrm{~h}$ at room temperature. Peptides were desalted on Ultra MicroTIP Columns (The Nest Group, Southborough, MA, USA) and dried in a SpeedVac concentrator. Dried peptides were resolubilized in $20 \mu \mathrm{L}$ HPLC grade water containing $0.1 \%$ formic acid. Sample analysis was performed on a linear ion trap LTQ mass spectrometer (Thermo Electron, San Jose, CA) equipped with a nanoelectrospray ion source (Thermo Electron) coupled to an Agilent 1100 micro HPLC system. Peptides were loaded with a cooled Agilent autosampler on a $2 \mathrm{~cm}$ long pre-column filled with C18 resin (Magic C18 AQ $5 \mu \mathrm{m}$; Michrom Bioresources, Auburn, CA, USA). A linear gradient of 80 minutes from $5 \%$ to $40 \%$ acetonitrile in $\mathrm{H}_{2} \mathrm{O}$ with $0.1 \%$ formic acid was used to separate peptides on a $10 \mathrm{~cm}$ long fused silica emitter packed with C18 resin spraying directly into the mass spectrometer at a flow rate of $0.5 \mu \mathrm{l} /$ $\mathrm{min}$. The MS instrument was operated in positive ion mode. The data-dependent acquisition mode was set to acquire one MS scan followed by three collision induced dissociation MS/MS scans. The MS full scans were recorded over a mass range of 400-1600 m/z. Dynamic exclusion was enabled, the repeat count was set to 2 and the exclusion duration to 30s. Further MS conditions were set as following: spray voltage 1.95 $\mathrm{kV}$, transfer capillary temperature $230^{\circ} \mathrm{C}$, normalized collision energy $35 \%$, activation q 0.25 and activation time $30 \mathrm{~ms}$.

The acquired raw files were converted to mzXML files using ReAdW with default settings and searched against the mouse IPI database version 3.26 with the Sequest search algorithm. The Sequest search parameters contained the static modification of cysteine +57.02 Da, at least one tryptic terminus and one missed cleavage was allowed. The data were further processed using the using the Trans-Proteomic Pipeline TPP including PeptideProphet and ProteinProphet to estimate the false discovery rate in the datasets. A protein probability of 0.5 was set as a cutoff corresponding to a false discovery rate of approximately $5 \%$. The protein list was annotated for secreted proteins using the algorithm SignalP and further manually curated for secreted proteins using UniProt database and literature search. Functional annotation was assigned using the PANTHER Classification system.

Relative enrichment or depletion in the CD44+CD24- population post-TGF- $\beta$ treatment was determined relative to mock-treated CD44+CD24- cells and data was normalized to GAPDH expression levels (relative 
change in GAPDH expression was 1.2 fold increase post TGF- $\beta$ treatment). Fold-changes (increase or decrease) above 5 was considered significant.

\section{Preparation of cell extracts}

Untreated or TGF- $\beta$ treated LNCaP and DU145 cells were washed twice in PBS (pH 7.4), scraped into $15-\mathrm{mL}$ conical tubes, and centrifuged at $1,000 \times \mathrm{g}$ at $4^{\circ} \mathrm{C}$ for 5 minutes. Cell extracts were prepared by lysis in NET buffer [50 mmol/L Tris-HCl, (pH 7.4), $150 \mathrm{mmol} / \mathrm{L} \mathrm{NaCl,} \mathrm{0.1 \%} \mathrm{NP40,} 1 \mathrm{mmol} / \mathrm{L}$ EDTA, 0.25\% gelatin, $0.02 \%$ sodium azide, $1 \mathrm{mmol} / \mathrm{L}$ phenylmethylsulfonyl fluoride, and $1 \%$ aprotinin]. The lysates were centrifuged at $15,000 \times \mathrm{g}$ for 30 minutes at $4^{\circ} \mathrm{C}$. The protein concentration of the supernatants was determined according to the method of Bradford using a Bio-Rad protein assay kit (Bio-Rad Laboratories, Richmond, CA).

\section{Western blot analysis}

Ten micrograms of total LNCaP or DU145 cell protein extracts were resolved by 10\% SDS-PAGE and transferred to nitrocellulose membranes. Membranes were blocked with 5\% nonfat dry milk in PBS/Tween $20(0.05 \%)$, followed by incubation with an anti-PCBP1 antibody (1:2000 dilution in 10\% milk/PBS-T). Post-exposure blots were stripped and probed with anti-GAPDH antibody (1:5000 dilution). The detection was done using horseradish peroxidase-labeled secondary antibodies and enhanced chemiluminescence detection reagent.

Overexpression of PCBP1

Human PCBP1 plasmid in pCMV6-AC-GFP backbone and the empty vector were obtained from Origene. CD24-CD44+ population isolated from LNCaP or DU145 cells $\left(4 \times 10^{5}\right)$ were transfected with the PCBP1 construct or the empty vector using Lipofectamine LTX (Life Technologies, Carlsbad, CA) according to the manufacturer's instructions. Forty-eight hours after transfection, cells were split 1:10 and subsequently selected with G418 (250 $\mu \mathrm{g} / \mathrm{ml}$ ) (Teknova, Hollister, CA) for approximately 2 weeks.

\section{Soft-agar assay}

Parental or PCBP1 overexpressing CD44+CD24- subset $\left(5 \times 10^{3}\right.$ cells per $35-\mathrm{mm}$ well) isolated from LNCaP or DU145 cells were resuspended in complete medium containing $0.35 \%$ agarose. Cells were grown on tissue culture dishes containing a 2-mL layer of solidified $0.7 \%$ agar in a complete medium. After 14 days, number of colonies was quantified from two randomly taken micrographs per well (original magnification, $\times 20$ ). For visualization, foci were methanol-fixed and stained with $0.005 \%$ crystal violet.

In vivo tumorigenesis assays

4 clones each of pCMV6-AC-GFP (vector control) and pCMV6-AC-PCBP1-GFP (PCBP1 overexpressing)) expressing CD44+CD24- population sorted from LNCaP or DU145 cells were resuspended in $50 \mu \mathrm{l}$ RPMI1640 or DMEM medium, respectively, and mixed with $50 \mu$ l Matrigel (Becton Dickinson) at a 1:1 ratio and held on ice. The entire $100 \mu \mathrm{l}$ sample ( $5 \times 10^{4}$ cells) was injected into each flank of 6-8 weeks old NOD/SCID mice anesthetized with isoflurane ( $n=5$ for each construct). Animals were accessed at the end of 4 weeks for the formation of tumors.

\section{Statistical analysis}

Statistical analysis was performed using SPSS 19.0 software. The results were expressed as mean \pm SD. The data were treated by Student's t test to determine statistical significance. $\mathrm{P}<0.05$ was considered statistically significant.

\section{Results}

CD44+CD24-population in LNCaP and DU145 cells are enriched following TGF- $\beta$ treatment

Since it has been previously suggested that TGF- $\beta$ can contribute to stemness of prostate cancer cells $[12,13]$, we sought to determine the effect of TGF- $\beta$ on the abundance of CD44+CD24- population in the LNCaP cell line. Fluorescent activated cell sorting (FACS) 
A

B

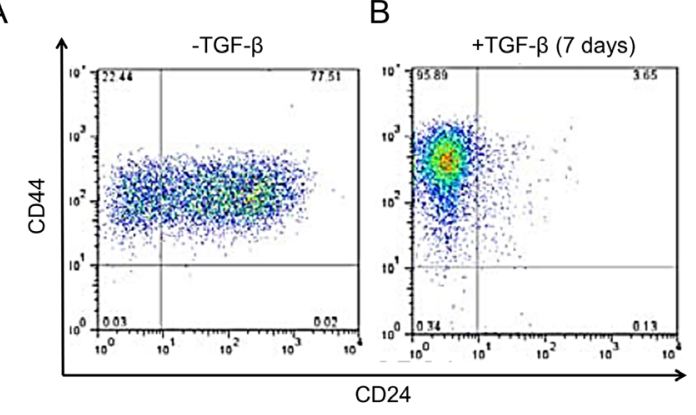

C

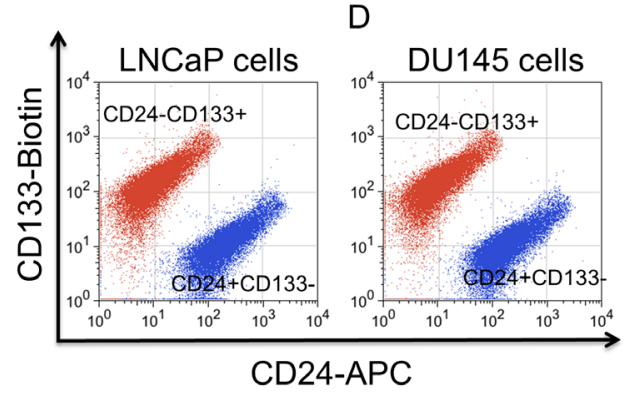

$\mathrm{E}$

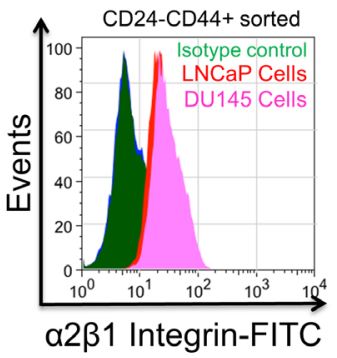

Fig. 1. TGF- $\beta$ induces enrichment of CD44+CD133+ $\alpha 2 \beta 1$ Integrin+CD24- population in LNCaP and DU145 cells. Flow cytometric analysis to assess CD24 and CD44 expression in untreated (A) and TGF- $\beta$-treated (B) LNCaP cells. Flow cytometric analysis of CD133 expression in CD44-CD24+ (shown in blue) and CD44+CD24- (shown in red) obtained from untreated or TGF- $\beta$-treated, respectively, LNCaP (C) and DU145 (D) cells. Shown are dot plots of CD24 and CD133 expression. (E) Assessment of $\alpha 2 \beta 1$ Integrin expression in CD44+CD24- cells sorted from TGF- $\beta$-treated LNCaP and DU145 cells. All data are representative of a minimum of three individual experiments.

revealed that CD44+CD24- were significantly enriched in LNCaP cells treated with TGF- $\beta$ for 7 days $(22.35 \pm 0.94 \%$ in mock treated vs $95.23 \pm 2.34 \%$ in TGF- $\beta$ treated cells; $P<0.01$ ) (Fig. $1 \mathrm{~A}, \mathrm{~B})$. The enrichment in CD44+CD24-population was accompanied by a significant depletion of CD44+CD24+ population during the same time span $(76.93 \pm 2.31 \%$ in mock treated vs $3.78 \pm 0.59 \%$ in TGF- $\beta$ treated cells; $\mathrm{P}<0.01$ ) (Fig. $1 \mathrm{~A}, \mathrm{~B}$ ). To confirm that this observation is not limited to one cell type we assessed the enrichment of CD44+CD24-population in another cell line, DU145 (Fig. 1C-E). In addition, we also assessed the expression levels of other prostate cancer stem cell makers, CD133 (Fig. 1C, D) and $\alpha 2 \beta 1$ Integrin (Fig. 1E). The CD24-CD44+ cell population obtained after 7 days of TGF- $\beta$ treatment were enriched for both CD133 and $\alpha 2 \beta 1$ Integrin in both LNCaP and DU145 cell lines (Fig. 1C-E). No significant CD133 expression was observed in the CD24+CD44- cell population in untreated LNCaP or DU145 cells (Fig. 1C, D; blue dots).

\section{PCBP1 is significantly downregulated in enriched CD44+CD24-population}

In order to determine the underlying mechanism driving enrichment of CD44+CD24population in LNCaP cells following TGF- $\beta$ treatment we performed proteomic profiling of CD44+CD24- cells isolated from mock or 7 days TGF- $\beta$ treated LNCaP cells. Following mass spectrometry, data analysis and normalization to GAPDH expression levels, 63 proteins showed either a 5 fold up- or down-regulation (Fig. 2A). Of these, 34 proteins were upregulated and 29 proteins were downregulated in the TGF- $\beta$-enriched CD44+CD24- cells. The protein that was maximally downregulated was poly $\mathrm{r}(\mathrm{C})$ binding protein-1 (PCBP1) or heterogeneous nuclear ribonucleoprotein (hnRNP)-E1 (9.31 \pm 0.05 folds) (Fig. 2A). We subsequently validated the steady state expression level of PCBP1 protein in CD44+CD24-cells isolated from LNCaP (Fig. 2B) and DU145 (Fig. 2C) cells for the indicated time points. PCBP1 


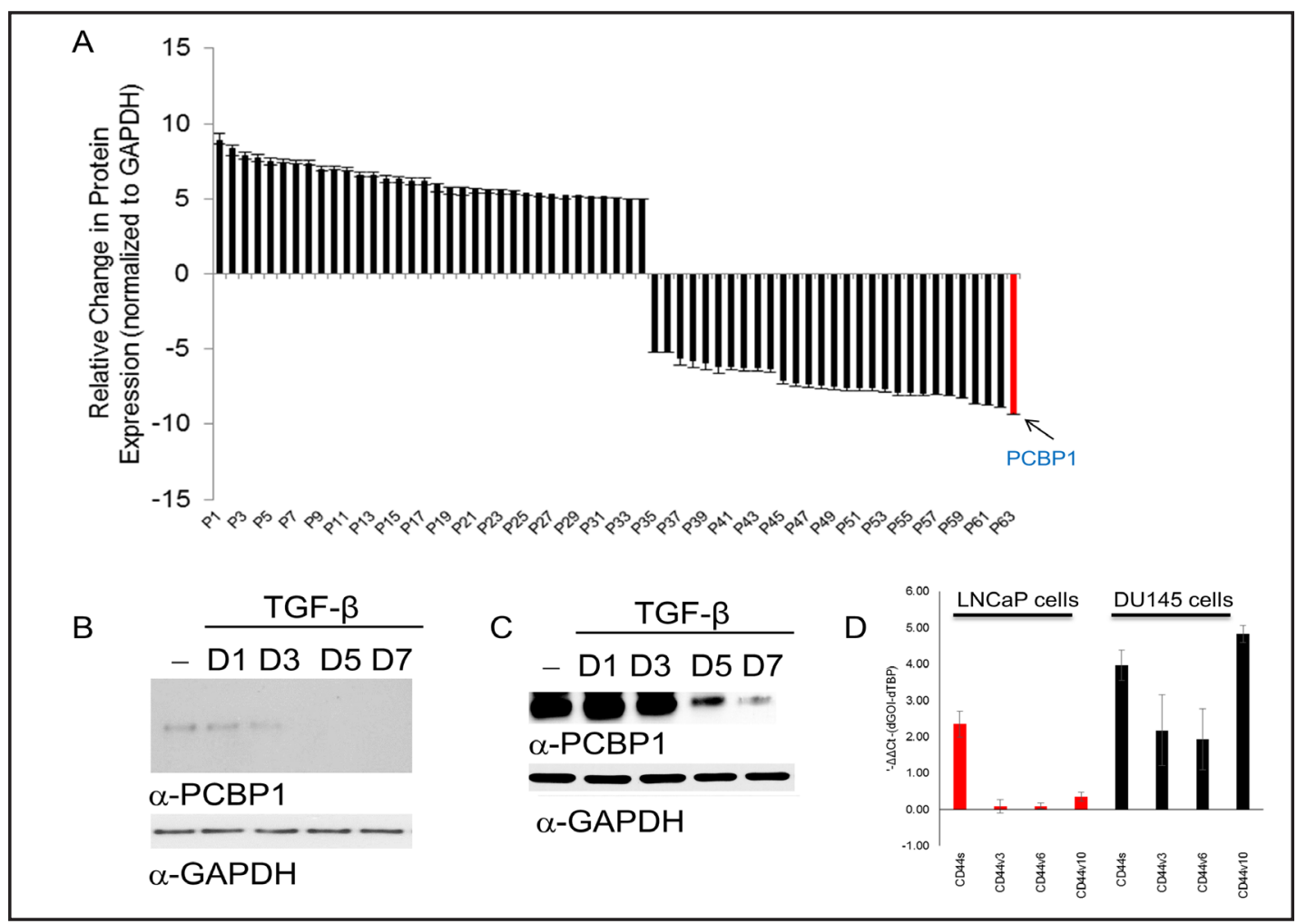

Fig. 2. TGF- $\beta$ induces downregulation of PCBP1 protein expression in enriched CD44+CD24- population isolated inform LNCaP and DU145 cells. (A) Observed fold changes of 63 proteins (representing at least 5 order of magnitude change) up- or down-regulated following TGF- $\beta$ treatment. The data represents experiment done in triplicate and post-normalization to GAPDH expression. (B, C) Immunoblot analysis of PCBP1 (upper panel) protein levels in CD44+CD24- cells isolated from LNCaP (B) and DU145 (C) cells treated with TGF- $\beta$ for the indicated time points. The blots were stripped and re-probed for GAPDH (bottom panel) as a loading control. (D) Steady state expression of $C D 44$ isoforms quantitated by qRT-PCR in stem cells enriched from LNCaP and DU145 cells.

protein expression was significantly downregulated after 5 days of TGF- $\beta$ treatment (Fig. 2B, C), further validating our proteomics data (Fig. 2A). Of note, the steady state expression level of PCBP1 was higher in DU145 cells compared to LNCaP cells. Cumulatively, our data suggest that PCBP1 is robustly downregulated when the CD44+CD133+ $22 \beta 1$ IntegrinCD24population is progressively enriched concomitant with prolonged TGF- $\beta$ treatment. Since LNCaP cells are largely considered CD44- [14], we evaluated expression of different CD44 isoforms in the CD44+CD133+ $\alpha 2 \beta 1$ IntegrinCD24- cell population sorted from LNCaP and DU145 cells (Fig. 2D). DU145 cells showed robust transcription of CD44s, CD44v3, CD44v6, and $C D 44 v 10$ in comparison to only CD44s expression in the LNCaP cells.

Overexpression of PCBP1 in either LNCaP or DU145 cells can attenuate enrichment of stem cell population following TGF- $\beta$ treatment

Our next objective was to determine if downregulation of PCBP1 is functionally required for observed enrichment of stem cell population in LNCaP and DU145 cells following TGF- $\beta$ treatment. For the same, we transfected a pCMV-AC-PCBP1-GFP plasmid into LNCaP and DU145 cells and generated both stable pools and clones for subsequent downstream experiments. Successful overexpression following stable selection was tested by fluorescence microscopy for GFP; both empty vector and PCBP1 overexpressing cells showed robust GFP expression in comparison to the mock transfected cells (Fig. 3A). Successful transfection was also confirmed via western blot (data not shown). FACS analysis revealed that CD44+CD24- 

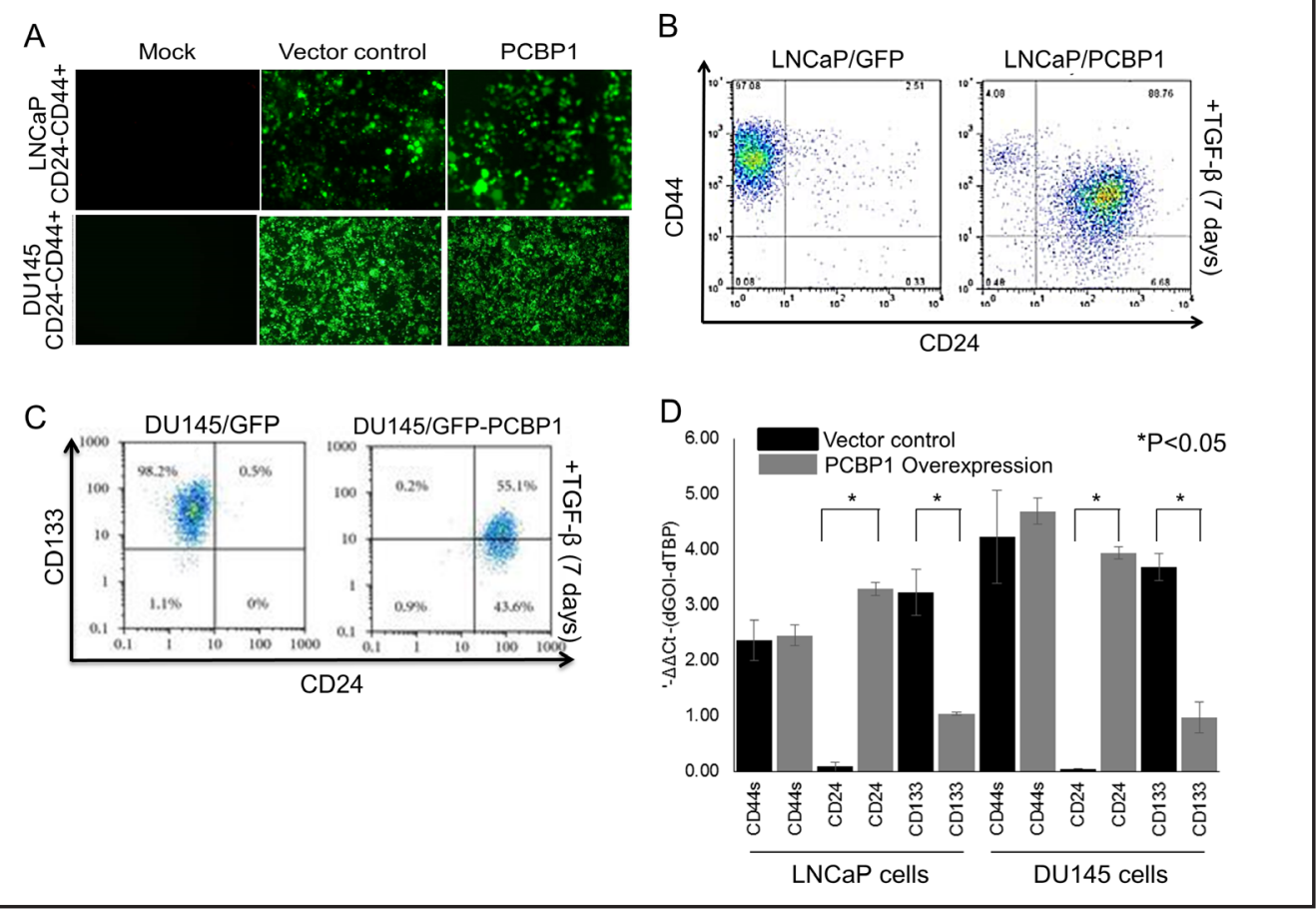

Fig. 3. Effect of PCBP1 protein overexpression on enrichment of CD44+CD24- population in LNCaP and DU145 cells following TGF- $\beta$ treatment. (A) Immunofluorescence images obtained from mock (left panel), pCMV6-AC-GFP (vector control) (middle panel), or pCMV6-AC-PCBP1-GFP (PCBP1) (right panel) transfected LNCaP (upper panels) and DU145 (lower panel) cells post stable selection with G418 (mock transfected cells were not selected). (B) Flow cytometric analysis of empty vector ( LNCaP/GFP) (left panel) and pCMV6AC-PCBP1-GFP transfected ( $L N C a P / P C B P 1$ ) (right panel) LNCaP cells post TGF- $\beta$-treatment for 7 days. Shown are GFP+ cells, which were in excess of $99 \%$ in each of the experiments conducted. (C) Flow cytometric analysis of empty vector (DU145/GFP) (left panel) and pCMV6-AC-PCBP1-GFP transfected (DU145/ GFP-PCBP1) (right panel) DU145 cells post TGF- $\beta$-treatment for 7 days. Shown are GFP+ cells, which were in excess of $99 \%$ in each of the experiments conducted. (D) Steady state expression of $C D 44, C D 24$, and $C D 133$ isoforms quantitated by qRT-PCR in stem cells enriched from LNCaP and DU145 cells. All data are representative of a minimum of three individual experiments. UTR, untranslated region.

were significantly enriched in LNCaP cells transfected with the empty vector (LNCaP/ GFP cells) post treatment with TGF- $\beta$ for 7 days (98.09 $\pm 4.29 \%$ ) (Fig. 3B), similar to what was observed initially (Fig. 1A, B). However, overexpression of PCBP1 (LNCaP/PCBP1) significantly attenuated the enrichment of CD44+CD24- population during the same time span, even after TGF- $\beta$ treatment for 7 days $(5.11 \pm 1.99 \%)(\mathrm{P}<0.001)$ (Fig. 3B). We also tested CD133 expression post overexpression of PCBP1. Whereas CD133+CD24- cells were significantly enriched in DU145 cells transfected with the empty vector (DU145/GFP cells) post treatment with TGF- $\beta$ for 7 days $(98.37 \pm 1.38 \%$ ), overexpression of $P C B P 1$ (DU145) GFP-PCBP1) significantly attenuated the enrichment of CD133+CD24+ population during the same time span, even after TGF- $\beta$ treatment for 7 days $(0.2 \pm 0.09 \%)(P<0.001)$ (Fig. 3C). Similar changes in CD133 expression was observed in LNCaP/PCBP1 cells (data not shown). However, loss of CD44 expression seemed to be more drastic than corresponding loss of CD133 expression (Fig. 3B, C and data not shown). Cumulatively, our data suggested that downregulation of PCBP1 protein expression is necessary for observed enrichment of CD44+CD133+CD24- stem cells in LNCaP and DU145 cell lines following TGF- $\beta$ treatment.

Quantitative real-time PCR (qRT-PCR) revealed that PCBP1 overexpression was correlated to significant $(\mathrm{P}<0.05)$ upregulation of $C D 24$ and significant $(\mathrm{P}<0.05)$ downregulation of $C D 133$ mRNA levels (Fig. 3D). No significant variation was observed in 
Fig. 4. Effect of PCBP1 protein overexpression on tumorigenicity of stem cells isolated from LNCaP and DU145 cells. (A) Relative number of soft agar colonies obtained from vector control (mock) or pCMV6-AC-PCBP1-GFP (PCBP1) transfected LNCaP (left) and DU145 (right) enriched stem cells post stable selection with G418. Data represents mean \pm SD of five independent experiments. (B) Percent of tumor formation observed 4 weeks after flank injection of vector control (mock clones) or PCBP1-overexpressing (PCBP1 clones) LNCaP stem cell clones into NOD/SCID mice. Each clone was injected in 5 animals. Data represents mean \pm SD of three independent experiments.

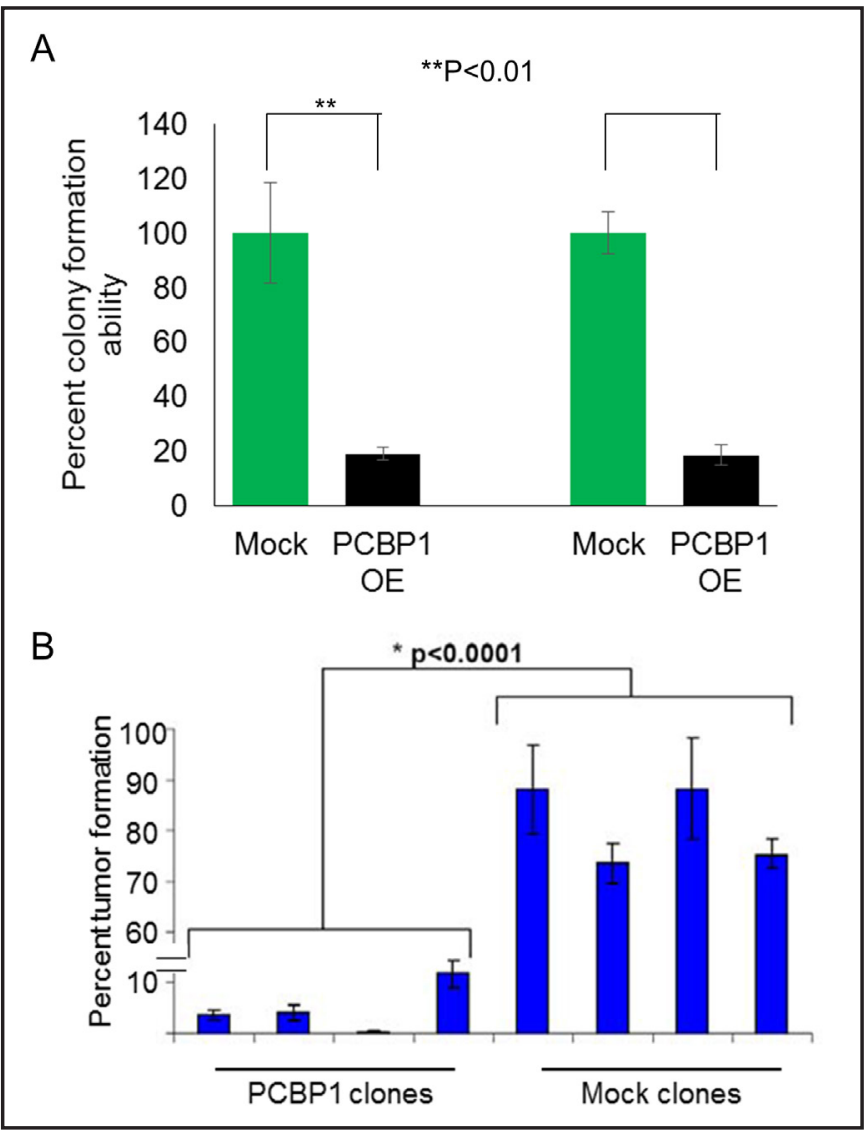

CD44 mRNA expression levels. Our results are indicative of PCBP1 potentiating signaling mechanisms that transcriptionally regulate CD24 and CD133 expression, which subsequently through an unknown mechanism downregulates CD44 expression.

Downregulation of PCBP1 protein expression is required for potent pro-tumorigenic activity observed in enriched CD44+CD24- population

In order to further determine the role of PCBP1 in regulating the stemness of LNCaP and DU145 cells, we performed soft agar colony formation assay using the mock transfected and PCBP1 overexpressing LNCaP and DU145 CD44+CD133+CD24- (stem) cells. As expected, TGF- $\beta$ treated parental LNCaP or DU145 stem cells formed soft agar colonies in a vast majority of the cases (96 $\pm 12 \%$ in LNCaP and $96.3 \pm 3.4 \%$ in DU145); in comparison, the PCBP1 overexpressing cells showed a significant decrease in colony formation $(18.3 \pm 1.83 \%$ in LNCaP and $19.2 \pm 2.3 \%$ in DU145; $\mathrm{P}<0.01$ in each case) (Fig. $4 \mathrm{~A}$ ). We furthermore tested the ability of the aforementioned stem cells to form tumors. Since stable pools can give false positives we used four different clones, each from empty vector and PCBP1 overexpressing LNCaP stem cells (Fig. 4B). Each clone was injected into flanks of five 6-8 weeks old NOD/SCID mice and tumor formation was compared four weeks after injection. After 4 weeks, distinctive tumors were observed in a vast majority of mice injected with LNCaP enriched stem cells carrying the empty vector $(81.39 \pm 8.96 \%)$. However, in mice injected with PCBP1 overexpressing LNCaP enriched stem cells, tumors formed in significantly less percentage of mice (5.93 $\pm 4.23 \%$; $\mathrm{P}<0.001$, compared to empty vector control group) (Fig. 4B). Not much heterogeneity was observed among the different clones tested in this assay. Cumulatively, our results showed that downregulation of PCBP1 is important for increase of stemness of LNCaP cells post TGFtreatment and is also perhaps central to tumorigenicity of these cells as revealed by lack of potent tumorigenic activity post-overexpression of PCBP1 in the LNCaP cells. 


\section{Discussion}

It has been widely proposed that both tumorigenesis and metastatic progression is contingent on transformation of a subpopulation of normal stem or progenitor cells, characterized by low expression of CD24 and high expression of CD44 [15-17]. The inherent properties of stem/progenitor cells may impart their transformed counterparts with the ability to evade traditional antitumor therapies and to establish metastasis [15-17]. Previous studies of CSCs and therapy resistance in other tumor systems have shown that CSCs appear to resist chemo- and radio- therapy, whereas therapy resistant cancer cells possess enhanced CSCs properties. Hence, it is pertinent to discover druggable mechanistic targets that drive the enrichment and maintenance of CSCs in prostate tumors.

Our proteomic profiling and subsequent validation indicate that PCBP1 is central to CSCs enrichment and functionality in prostate cancer. To the best of our knowledge this is the first report of any direct regulator of CSC enrichment in prostate cancer. LNCaP cells were originally classified as CD44 negative through global studies involving multiple prostate cancer cell lines and allocating cut-off values to protein expression and calling negative if expression of a protein did not meet the cut-off. But this really did not indicate complete absence of the secreted product. It must be noted that such experiments were conducted in whole cell lysates prepared from the entire population of LNCaP cells and not any subset population as in the current case.

It has been recently shown that PCBP1 regulates the stability of the pro-oncogenic and pro-stemness p63 transcript in pancreatic, ovarian and breast cancer cell line [18]. However, in the aforementioned context PCBP1 downregulation caused p63 degradation, which would suggest that PCBP1 is a positive regulator of stemness, in apparent contradiction to our current findings. However, two separate studies corroborate our findings. In one of those, a transcript-selective translational regulatory pathway was described in which a ribonucleoprotein (mRNP) complex, consisting of Poly(C) binding protein (PCBP) 1, silences translation of Dab2 and ILEI mRNAs, which are involved in mediating EMT [19]. It was shown that TGF $\beta$ activates a kinase cascade terminating in the phosphorylation of PCBP1 by isoformspecific stimulation of protein kinase B $\beta / A k t 2$, inducing the release of the mRNP complex from the 3'-UTR element, in turn resulting in the reversal of translational silencing and increased expression of transcripts that mediates EMT [19-21]. In the second study, PCBP1 was shown to downregulate production of the pro-metastatic PRL-3 phosphatase [22]. Thus it might be possible that downregulation of PCBP1 dictates stemness and mesenchymal cell formation in a context dependent fashion, as observed by us in the current study and the others $[19,22]$.

It would be interesting to identify what potentially causes downregulation of PCBP1 following TGF- $\beta$ treatment in these cells. Our preliminary results suggest that it is not at the level of mRNA transcript and is perhaps being mediated by ubiquitin-mediated degradation of PCBP1 following TGF- $\beta$ treatment (data not shown). Another interesting objective would be to determine if a similar mechanism is operative in the sustained enrichment of CSCs in other tumor types.

Our current study had some potential limitations including the study being conducted in cell line rather than primary tumor cells and that the study was based on proteomic profiling in only one androgen sensitive prostate tumor cell line. Obviously it is difficult to do such a scale of experiments in primary tumor samples. However, further validation in a large number of prostate cancer cell lines is warranted and which is currently being conducted.

\section{Disclosure Statement}

None. 
Chen et al.: PCBP1 in Androgen Dependent Prostate Tumors

\section{Acknowledgements}

This work was granted by Program of Shanghai City Committee of Science and technology (No. 134119a9800) and National Science Foundation of China (81172450 and 81202008).

\section{References}

1 Clarke MF, Dick JE, Dirks PB, Eaves CJ, Jamieson CH, Jones DL, Visvader J, Weissman IL, Wahl GM: Cancer stem cells--perspectives on current status and future directions: AACR Workshop on cancer stem cells. Cancer Res 2006;66:9339-9344.

2 Tang DG, Patrawala L, Calhoun T, Bhatia B, Choy G, Schneider-Broussard R, Jeter C: Prostate cancer stem/ progenitor cells: identification, characterization, and implications. Mol Carcinogen 2007;46:1-14.

- Visvader JE, Lindeman GJ: Cancer stem cells in solid tumours: accumulating evidence and unresolved questions. Nat Rev Cancer 2008;8:755-768.

-4 Rosen JM, Jordan CT: The increasing complexity of the cancer stem cell paradigm. Science 2009;324:16701673.

5 Dalerba P, Dylla SJ, Park IK, Liu R, Wang X, Cho RW, Hoey T, Gurney A, Huang EH, Simeone DM, Shelton AA, Parmiani G, Castelli C, Clarke MF: Phenotypic characterization of human colorectal cancer stem cells. Proc Natl Acad Sci U S A 2007;104:10158-10163.

6 Yin AH, Miraglia S, Zanjani ED, Almeida-Porada G, Ogawa M, Leary AG, Olweus J, Kearney J, Buck DW: AC133, a novel marker for human hematopoietic stem and progenitor cells. Blood 1997;90:5002-5012.

7 Collins AT, Berry PA, Hyde C, Stower MJ, Maitland NJ: Prospective identification of tumorigenic prostate cancer stem cells. Cancer Res 2005;65:10946-10951.

8 Patrawala L, Calhoun T, Schneider-Broussard R, Li H, Bhatia B, Tang S, Reilly JG, Chandra D, Zhou J, Claypool K, Coghlan L, Tang DG: Highly purified CD44+ prostate cancer cells from xenograft human tumors are enriched in tumorigenic and metastatic progenitor cells. Oncogene 2006;25:1696-1708.

-9 Patrawala L, Calhoun T, Schneider-Broussard R, Zhou J, Claypool K, Tang DG: Side population is enriched in tumorigenic, stem-like cancer cells, whereas ABCG2+ and ABCG2- cancer cells are similarly tumorigenic. Cancer Res 2005;65:6207-6219.

10 Patrawala L, Calhoun-Davis T, Schneider-Broussard R, Tang DG: Hierarchical organization of prostate cancer cells in xenograft tumors: the CD44+alpha2beta1+ cell population is enriched in tumor-initiating cells. Cancer Res 2007;67:6796-6805.

11 Li H, Jiang M, Honorio S, Patrawala L, Jeter CR, Calhoun-Davis T, Hayward SW, Tang DG: Methodologies in assaying prostate cancer stem cells. Methods Mol Biol 2009;568:85-138.

12 Zhang T, Lee YW, Rui YF, Cheng TY, Jiang XH, Li G: Bone marrow-derived mesenchymal stem cells promote growth and angiogenesis of breast and prostate tumors. Stem Cell Res Ther 2013;4:70.

13 Ye H, Cheng J, Tang Y, Liu Z, Xu C, Liu Y, Sun Y: Human bone marrow-derived mesenchymal stem cells produced TGFbeta contributes to progression and metastasis of prostate cancer. Cancer Invest 2012;30:513-518.

14 Dhir R, Gau JT, Krill D, Bastacky S, Bahnson RR, Cooper DL, Becich MJ: CD44 Expression in Benign and Neoplastic Human Prostates. Mol Diagn 1997;2:197-204.

15 Reya T, Morrison SJ, Clarke MF, Weissman IL: Stem cells, cancer, and cancer stem cells. Nature 2001;414:105-111.

16 Behbod F, Rosen JM: Will cancer stem cells provide new therapeutic targets? Carcinogenesis 2005;26:703711.

17 Dean M, Fojo T, Bates S: Tumor stem cells and drug resistance. Nat Rev Cancer 2005;5:275-284.

18 Cho SJ, Jung YS, Chen X: Poly (C)-Binding Protein 1 Regulates p63 Expression through mRNA Stability. PLoS One 2013;8:e71724.

19 Chaudhury A, Hussey GS, Ray PS, Jin G, Fox PL, Howe PH: TGF-beta-mediated phosphorylation of hnRNP E1 induces EMT via transcript-selective translational induction of Dab2 and ILEI. Nat Cell Biol 2010;12:286293.

-20 Hussey GS, Chaudhury A, Dawson AE, Lindner DJ, Knudsen CR, Wilce MC, Merrick WC, Howe PH: Identification of an mRNP complex regulating tumorigenesis at the translational elongation step. Mol Cell 2011;41:419-431.

21 Hussey GS, Link LA, Brown AS, Howley BV, Chaudhury A, Howe PH: Establishment of a TGF $\beta$-induced posttranscriptional EMT gene signature. PLoS One2012;7:e52624.

22 Wang H, Vardy LA, Tan CP, Loo JM, Guo K, Li J, Lim SG, Zhou J, Chng WJ, Ng SB, Li HX, Zeng Q: PCBP1 suppresses the translation of metastasis-associated PRL-3 phosphatase. Cancer Cell 2010;18:52-62. 